\title{
Subacute effects of a maximal exercise bout on endothelium-mediated vasodilation in healthy subjects
}

\section{K. Bousquet-Santos ${ }^{1,3}$, P.P.S. Soares ${ }^{1}$ and A.C.L. Nóbrega ${ }^{1,2,3}$}

\author{
'Departamento de Fisiologia e Farmacologia, Instituto Biomédico, \\ ${ }^{2}$ Pós-Graduação em Ciências Cardiovasculares, \\ Universidade Federal Fluminense, Niterói, RJ, Brasil \\ 3Pós-Graduação em Fisiopatologia Clínica e Experimental, \\ Universidade do Estado do Rio de Janeiro, Rio de Janeiro, RJ, Brasil
}

\section{Correspondence \\ A.C.L. Nóbrega \\ Departamento de Fisiologia e \\ Farmacologia \\ Rua Professor Hernani Melo, 101 \\ 24210-130 Niterói, RJ \\ Brasil \\ Fax: +55-21-2629-2405 \\ E-mail: anobrega@urbi.com.br}

Presented at the VIII Brazilian Symposium on Cardiovascular Physiology, Louveira, SP, Brazil, June 4-6, 2004

Research supported by CNPq (No. 350553/1995-5), by a CAPES pre-doctoral scholarship, and by FAPERJ (No. E-26/170.505/2001).

Received July 29, 2004

Accepted January 14, 2005

\section{Abstract}

We evaluated vascular reactivity after a maximal exercise test in order to determine whether the effect of exercise on the circulation persists even after interruption of the exercise. Eleven healthy sedentary volunteers (six women, age $28 \pm 5$ years) were evaluated before and after $(10,60$, and $120 \mathrm{~min})$ a maximal exercise test on a treadmill. Forearm blood flow (FBF) was measured by venous occlusion plethysmography before and during reactive hyperemia $(\mathrm{RH})$. Baseline FBF, analyzed by the area under the curve, increased only at $10 \mathrm{~min}$ after exercise $(\mathrm{P}=0.01)$. FBF in response to $\mathrm{RH}$ increased both at 10 and 60 min $v$ s baseline $(\mathrm{P}=0.004)$. Total excess flow for $\mathrm{RH}$ above baseline showed that vascular reactivity was increased up to $60 \mathrm{~min}$ after exercise (mean \pm SEM, before: $526.4 \pm 48.8 ; 10$ min: $1053.0 \pm 168.2$;

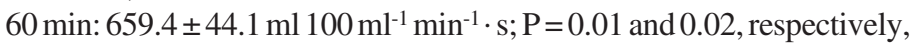
$v s$ before exercise). The changes in FBF were due to increased vascular conductance since mean arterial blood pressure did not change. In a time control group ( $N=5,34 \pm 3$ years, three women) that did not exercise, FBF and RH did not change significantly $(\mathrm{P}=0.07$ and 0.7 , respectively). These results suggest that the increased vascular reactivity caused by chronic exercise may result, at least in part, from a summation of the subacute effects of successive exercise bouts.

\section{Introduction}

Exercise training reduces overall morbidity and mortality in the general population (1) and in subjects with established cardiovascular disease (2). Although the mechanisms involved in this protective effect are complex and not completely understood, improved vascular function, i.e., increased vasodilatory reactivity, has been ob-
Key words - Vascular reactivity

- Exercise test

- Blood flow

- Plethysmography 
of the vascular endothelium plays a key role in all stages of atherogenesis, from lesion initiation to plaque rupture (6).

Regular aerobic exercise can both prevent the age-associated loss in endotheliumdependent vasodilation and restore the function lost as a result of sedentary aging (5). Twelve weeks of moderate exercise, but not mild- or high-intensity exercise, increased endothelium-dependent vasodilation through increased nitric oxide production and release in healthy subjects (7). The chronic effects of exercise develop from the interaction of physiological responses that occur during (acute effects) and after each session of physical effort (subacute effects). Therefore, it is important to evaluate the subacute effects of exercise to understand the whole process of adaptation. However, little is known about the physiological phenomenon that occurs after a single session of physical exertion. Baynard et al. (8) demonstrated that endurance-trained men appear to have a greater peak vasodilatory capacity compared to resistance-trained men, and acute maximal exercise on a treadmill increased the vasodilatory capacity, measured only once after physical effort, in both groups. Copeland et al. (9) observed increases in forearm blood flow (FBF) after acute handgrip exercise, and these increases were greater in the aerobically trained than in the resistance-trained individuals. It is not known whether the subacute effects of exercise on vascular reactivity also occur in healthy sedentary subjects and, if they actually occur, for how long after exercise they are sustained.

On the basis of these considerations, the purpose of the present study was to determine peripheral blood flow and vascular reactivity for $2 \mathrm{~h}$ after a single bout of maximal aerobic exercise in healthy subjects.

\section{Subjects and Methods}

Eleven healthy subjects (six women; mean age $28 \pm 5$ years) volunteered to participate in the study, which was approved by the Institutional Ethics Committee (CEP CCM/ HUAP 030/04). All subjects gave written informed consent to participate in the study. Exclusion criteria were the presence of diabetes, hypertension, known cardiovascular disease, smoking habit, and the use of medications. The subjects fasted for at least $2 \mathrm{~h}$ and did not perform vigorous exercise in the preceding $24 \mathrm{~h}$ or ingested caffeine on the day of the evaluation. All tests were performed in the morning, after a 20 -min period of supine rest in a quiet, air-conditioned room (constant temperature of $23^{\circ}$ to $25^{\circ} \mathrm{C}$ ).

FBF was measured with a mercury-filled silastic strain-gauge plethysmograph (EC6, D.E., Hokanson, Inc., Bellevue, WA, USA). A cuff was placed around the upper arm, a strain gauge around the widest part of the right forearm and an additional cuff around the wrist to occlude hand circulation. The arm was supported by a device at the level of the heart. One minute before each measurement and throughout FBF measurement, the wrist cuff was inflated to $200 \mathrm{mmHg}$. FBF was determined by rapidly inflating the upper cuff to $40 \mathrm{mmHg}$ for $10 \mathrm{~s}$ to occlude venous circulation, followed by a 10 -s deflation, during each 20-s cycle. An average of nine 20-s plethysmographic cycles were used for resting FBF measured as the rate of volume change during venous occlusion and expressed as $\mathrm{ml} / 100 \mathrm{ml}$ of forearm tissue volume/min. Reactive hyperemia (RH) was induced by inflating the upper arm cuff to $200 \mathrm{mmHg}$ for $5 \mathrm{~min}$, after which the cuff was deflated for $10 \mathrm{~s}$ and FBF measured for 3 min thereafter as described above. The area under the curve (AUC) both at baseline and during $\mathrm{RH}$ was calculated as a flow-time index to provide values of total FBF in each situation. Vascular reactivity was considered to be the total excess flow above baseline, calculated as the difference between the AUC during RH and at baseline. The plethysmographic signal was transmitted to the computer using an A/D conversion board 
(National Instruments, Co., Autix, TX, USA) for off-line analysis. All data analyses were performed by the same investigator, who showed an intra-subject variability (coefficient of variation) of 1.4 and $1.8 \%$ for resting and RH measurements, respectively. Heart rate and blood pressure were monitored using the digital infrared photoplethysmographic Finapres device (model 2050, Ohmeda, Englewood, CO, USA) and mean arterial pressure (MAP) was calculated by the standard equation: MAP $=$ diastolic blood pressure + (systolic blood pressure - diastolic blood pressure/3).

After baseline evaluation, subjects performed an exercise test on a treadmill (Inbramed, Porto Alegre, RS, Brazil) following a progressive, maximal intensity, and individualized ramp protocol that was designed to last approximately $10 \mathrm{~min}$, based on the subject's age and gender. The volunteers performed the procedures exactly as described above (resting FBF and RH) 10, 60 , and $120 \mathrm{~min}$ after completing the exercise test. To assure that the actual effects of exercise were observed, an additional timecontrol group was used $(\mathrm{N}=5)$. All individuals participated in the exercise experiment that was performed at least one week before the time-control test. However, due to technical limitations, data for two individuals were excluded from the exercise experiment. The same procedures were done as in the exercise test day except that, after the baseline values were recorded, the subjects stood for $10 \mathrm{~min}$ on the treadmill, with no movements, and the 10-, 60-, and 120-min evaluations were performed thereafter.

Results were analyzed statistically by oneway analysis of variance (ANOVA) for repeated measures. When ANOVA revealed significant differences, the post hoc Bonferroni or Dunnett test was used to determine which specific time points were different from pre-exercise data taken as reference. Data are reported as means \pm SEM. Statistical significance was set at $\mathrm{P}<0.05$.

\section{Results}

The demographic and exercise-related results for the exercise and control group are shown in Table 1.

The AUC for baseline FBF and RH for the different intervals of analysis are shown in Figure 1. Compared to pre-exercise values, baseline FBF (upper panel) was significantly increased at $10 \mathrm{~min}(\mathrm{P}=0.019)$, but not at $60 \mathrm{~min}$ or $120 \mathrm{~min}$ after exercise. FBF during RH (Figure 1, lower panel) was higher at $10 \min (\mathrm{P}=0.013)$ and $60 \min (\mathrm{P}=0.05)$

Table 1. Demographic and exercise-related results.

\begin{tabular}{lcc}
\hline & $\begin{array}{c}\text { Exercise group } \\
(\mathrm{N}=9)\end{array}$ & $\begin{array}{c}\text { Control group } \\
(\mathrm{N}=5)\end{array}$ \\
\hline $\begin{array}{lcc}\text { Age (years) } \\
\text { Gender (female) }\end{array}$ & $28 \pm 2$ & $34 \pm 3$ \\
Weight (kg) & $69.4 \pm 5$ & $65.9 \pm 4$ \\
Height (cm) & $1.73 \pm 0.03$ & $1.71 \pm 0.02$ \\
$\begin{array}{l}\text { Exercise time } \\
\text { (min) }\end{array}$ & $13.5 \pm 0.7$ & - \\
METs & $12.8 \pm 0.6$ & - \\
\hline
\end{tabular}

Data are reported as mean \pm SEM. METs = metabolic equivalents.
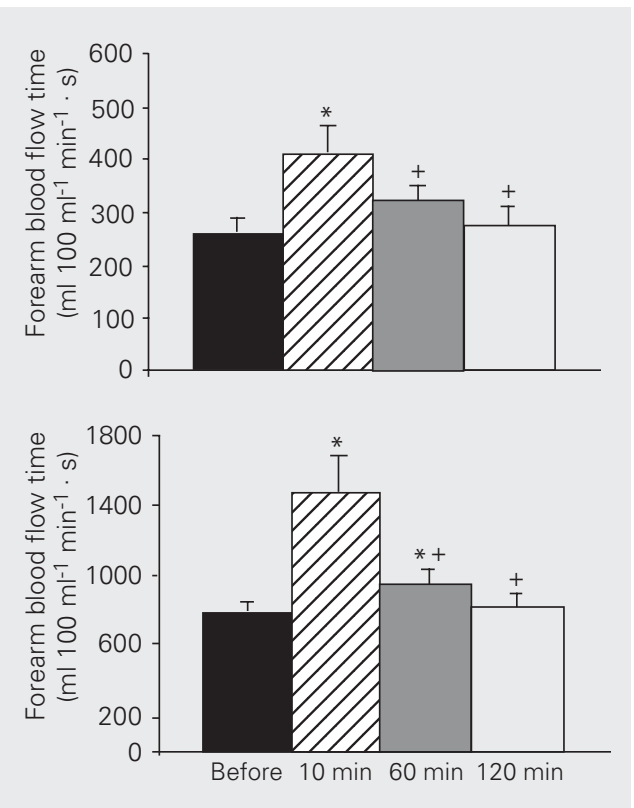

Figure 1. Forearm blood flow during baseline and reactive hyperemia calculated as the area under the curve (AUC). AUC for flow vs time was measured before and after the exercise test. Flow was higher for the baseline measurement only at 10 min after physical effort (upper panel). Values for reactive hyperemia were higher at 10 and $60 \mathrm{~min}$ after exercise (lower panel). ${ }^{*} \mathrm{P}<0.05$ vs before exercise; $+P<0.05$ vs 10 min after exercise (ANOVA for repeated measures with the post hoc Bonferroni test). 
after exercise and returned to values close to baseline at $120 \mathrm{~min}(\mathrm{P}>0.05)$. There was no significant correlation between FBF or HR and the peak exercise intensity achieved during the test.

Analysis of the whole temporal response to $\mathrm{RH}$ during the 3-min interval of recording (Figure 2, upper panel) showed that FBF was
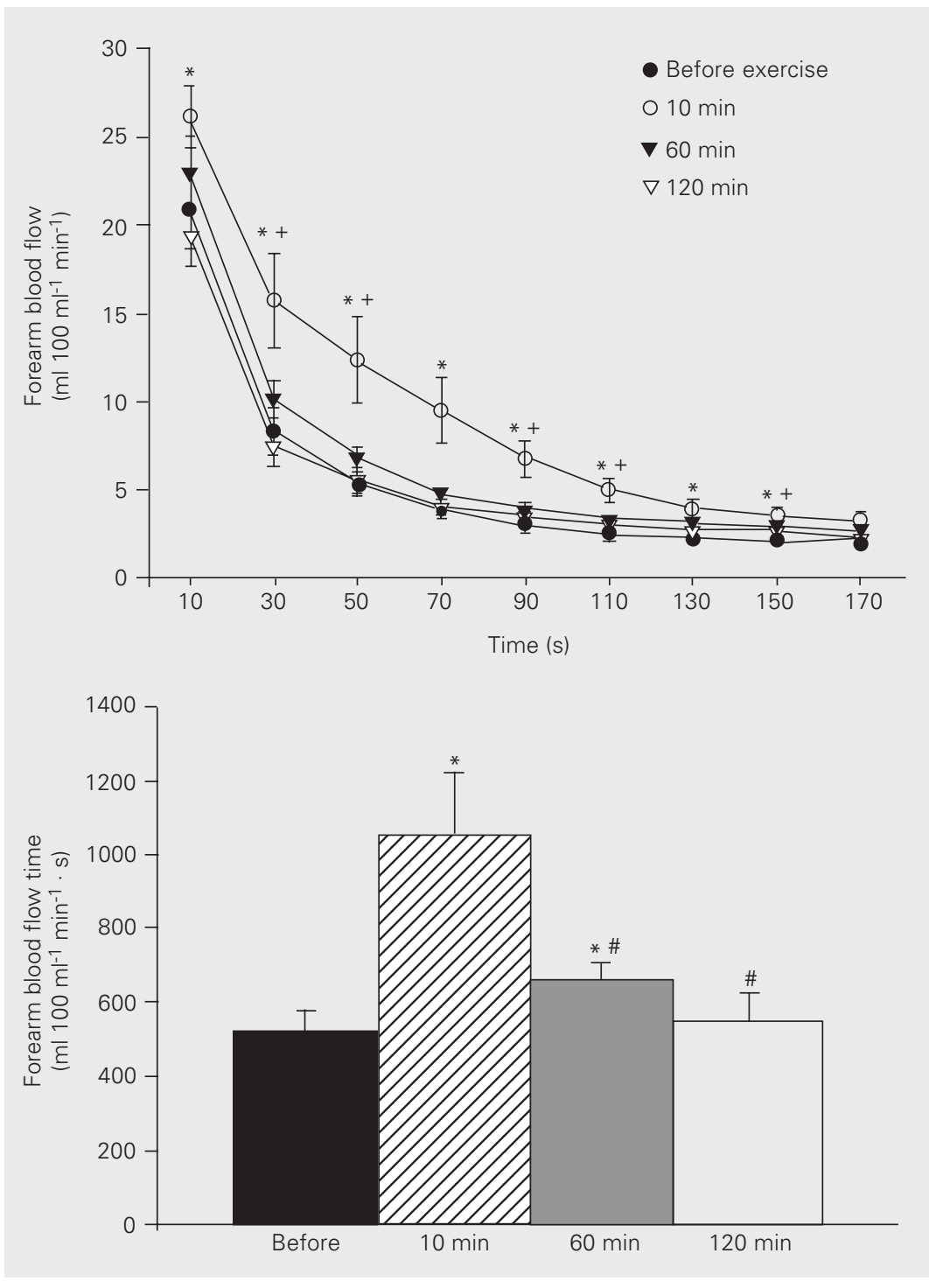

Figure 2. Temporal response and total excess blood flow during reactive hyperemia. Forearm blood flow increased during reactive hyperemia at $10 \mathrm{~min}$ and $60 \mathrm{~min}$ after the exercise test compared to before exercise (upper panel). Total excess flow (blood flow during reactive hyperemia minus baseline flow; lower panel), an indicator of vascular reactivity, was increased up to $1 \mathrm{~h}$ after cessation of exercise. ${ }^{*} \mathrm{P}<0.05$ for $10 \mathrm{~min}$ vs before exercise; $+P<0.05$ for 60 min vs before exercise; ${ }^{P} P<0.05$ vs 10 min (ANOVA for repeated measures with the post hoc Bonferroni test). increased throughout all but the last measurements obtained at $10 \mathrm{~min}$ post-exercise compared to the pre-exercise. FBF was also increased $60 \mathrm{~min}$ after the exercise test at several time points (Figure 2, upper panel). When vascular reactivity was analyzed as AUC of excess flow (Figure 2, lower panel), exercise caused an increase at $10 \mathrm{~min}(\mathrm{P}=$ $0.015)$ and $60 \min (\mathrm{P}=0.02)$, but not at 120 $\min$.

There were no significant changes in MAP in the 10-min post-exercise period or at the other times (data not shown). Therefore, the subacute flow changes provoked by exercise were due to increases in vascular conductance and not simply to higher driving pressure.

When the results of the time-control group were evaluated, a progressive decrease in baseline FBF and RH was observed although without statistically significant changes. While the baseline FBF values increased by $73 \%$ in the post-exercise interval for the experimental group, the time control group showed a decrease of 15 to $25 \%$ at the $10-$, 60-, and 120-min measurements compared to the pre-exercise values (data not shown). For the $\mathrm{RH}$ analysis, the decrease over time was of $11-21 \%$, contrasting to the $25 \%$ increase in the 10-min post-exercise interval in the experimental group. These findings reflect the effect of time on blood flow measurements and served as an internal control to support the view that the enhanced blood flow found in the experimental group was due to the physical test stimulus.

\section{Discussion}

The main finding of the present study was that an acute maximal aerobic exercise test enhances baseline FBF and vascular reactivity during RH. Vascular reactivity was still increased 60 min after the end of exercise. Although many studies have investigated the chronic adaptations to exercise training, little is known about the acute, and 
more specifically the subacute effects of a single bout of exercise on the vascular reactivity of healthy sedentary subjects.

The chronic effects of exercise training on endothelial function and vascular reactivity have been studied extensively. Eight weeks of combined aerobic and resistance training enhanced the vasodilation in response to RH and infusion of acetylcholine into individuals with type 2 diabetes (4). In healthy individuals, Maeda et al. (10) showed increased levels of plasma nitrite/nitrate and decreased levels of endothelin-1 after eight weeks training on a leg ergometer. Interestingly, this effect lasted until the 4th week after cessation of exercise training and the levels of nitric oxide and endothelin-1 returned to basal levels (before training) in the 8th week after completion of exercise training. These data suggest that the chronic effects of physical training develop over the weeks as an adaptation to the acute stress of exercise bouts.

Exercise acutely reduces triglycerides (11), increases HDL-cholesterol (11) as well as insulin sensitivity and ability to resynthetize the glycogen of skeletal muscle (12), and produces an acute blood pressure reduction (13). In fact, these effects could be classified as subacute since the reduction of triglycerides is not immediate but occurs 18$24 \mathrm{~h}$ after exercise and lower levels of blood pressure may persist for $24 \mathrm{~h}$ (14). Baynard et al. (8) investigated the vasodilatory response of endurance- and resistance-trained individuals before and after an acute bout of maximal aerobic exercise. The findings included a greater ability to respond to $\mathrm{RH}$ in endurance-trained individuals and a larger vasodilatory response following $\mathrm{RH}$, upon completion of acute exercise, in both endurance- and resistance-trained men. However, the authors evaluated the vasodilatory capacity in a physically active population and just before and immediately after the execution of physical exercise on a treadmill. More importantly, Baynard et al. (8) did not report the baseline blood flow after exercise. Thus, it is not possible to determine whether blood flow increased during $\mathrm{RH}$ because of an enhanced reactive vasodilation or because of increased enhanced overall vascular conductance.

In the present study, we evaluated the vasodilatory response of healthy sedentary individuals before and 10, 60 and $120 \mathrm{~min}$ after an exercise test on a treadmill. In agreement with other studies, we observed higher $\mathrm{FBF}$ at baseline and in response to $\mathrm{RH}$ in the post-exercise period (15). When the results were analyzed using the AUC, baseline values post-exercise were still higher compared to pre-exercise but an increased $\mathrm{RH}$ response could be detected not only in the post-exercise interval but also after $60 \mathrm{~min}$ of cessation of exercise test. In addition to the increased peak vasodilatory flow during $\mathrm{RH}$ immediately after the exercise test, we observed that the values obtained for the temporal response to RH maintained upper levels for most of the measurement period compared to the pre-exercise evaluation. Again, this profile was observed in the 60 -min postexercise measurement. Baynard et al. (8) showed a biphasic FBF response to $\mathrm{RH}$ following an acute exercise bout. After the initial $30 \mathrm{~s}$ after cuff deflation, FBF was exaggerated for about $1 \mathrm{~min}$, followed by a gradual decline towards baseline values, and this response was especially evident in the endurance-trained group.

An original finding of our study was that, although baseline values returned to the normal range in the 60-min post-exercise period, an increased vascular reactivity was observed, reflected by higher values of total excess flow. It may be assumed that the maximal exercise test evoked a subacute effect of increased vascular reactivity and increased vascular conductance, as the MAP did not change in the post-exercise period. Several mechanisms have been suggested to clarify the physiological processes involving enhanced subacute vasodilatory re- 
sponses mediated by exercise. It has been demonstrated that the increased post-occlusion brachial artery vasodilation observed after ischemic handgrip exercise is at least partly mediated by nitric oxide since infusion of L-NMMA, a nitric oxide synthase inhibitor, evoked lower post-occlusion vasodilation compared with infusion of $\mathrm{NaCl}$ (16). Joyner et al. (17) investigated the separate and combined contribution of nitric oxide and vasodilating prostaglandins as mediators of RH in the human forearm and observed that L-NMMA reduced baseline FBF at rest and had a modest effect on peak forearm vascular conductance and flow. Ibuprofen, a cyclooxygenase inhibitor, did not change FBF at rest and reduced peak FBF. When L-NMMA was infused after ibuprofen, a reduced FBF and no effect on peak flow was observed, suggesting that nitric oxide synthase inhibition has a modest effect on peak vasodilation during $\mathrm{RH}$ in contrast to the prostaglandins. Another potential mechanism for the increased vascular conductance after exercise is a diminished sympathetic activity leading to lower vasoconstrictor tone, as previously shown (18).
This could increase overall vascular conductance, but it is unlikely that decreased sympathetic vasoconstriction would cause a specific effect on vascular reactivity.

To exclude the temporal influence on the vasodilatory response, a time control group was submitted to the same protocol except for the exercise test. A progressive decrease in the values of baseline FBF and RH response was observed, which contrasted with the higher levels of those parameters observed in the group that performed exercise. This confirmed the subacute effect of the exercise test on the vasodilatory response.

In conclusion, a maximal aerobic exercise test provoked a subacute effect on vascular function that lasted $60 \mathrm{~min}$, with increased vascular conductance and vasodilatory reactivity in healthy young subjects.

\section{Acknowledgments}

We would like to thank Fabricia Junqueira das Neves, Marcio Oliveira Ramos, and Sonia Prado for their assistance during pilot experiments and with the preparation of the manuscript.

\section{References}

1. Wei M, Kampert JB, Barlow CE, Nichaman MZ, Gibbons LW, Paffenbarger Jr RS \& Blair SN (1999). Relationship between low cardiorespiratory fitness and mortality in normal-weight, overweight, and obese men. Journal of the American Medical Association, 16: 1547-1553.

2. Lee CD \& Blair SN (2002). Cardiorespiratory fitness and stroke mortality in men. Medicine and Science in Sports and Exercise, 34: 592-595.

3. Maiorana A, O'Driscoll G, Dembo L, Cheetham C, Goodman C, Taylor R \& Green D (2000). Effect of aerobic and resistance exercise training on vascular function in heart failure. American Journal of Physiology, 279: H1999-H2005.

4. Maiorana A, O'Driscoll G, Cheetham C, Dembo L, Stanton K, Goodman C, Taylor R \& Green D (2001). The effect of combined aerobic and resistance training on vascular function in type 2 diabetes. Journal of the American College of Cardiology, 38: 860-866.

5. DeSouza CA, Shapiro LF, Clevenger CM, Dinenno FA, Monahan KD, Tanaka H \& Seals DR (2000). Regular aerobic exercise prevents and restores age-related declines in endothelium-dependent vasodilation in healthy men. Circulation, 102: 1351-1357.
6. Behrendt D \& Ganz P (2002). Endothelial function: from vascular biology to clinical applications. American Journal of Cardiology, 90: $40 \mathrm{~L}-48 \mathrm{~L}$.

7. Goto C, Higashi Y, Kimura M, Noma K, Hara K, Nakagawa K, Kawamura M, Chayama K, Yoshizumi M \& Nara I (2003). Effect of different intensities of exercise on endothelium-dependent vasodilation in humans: role of endothelium-dependent nitric oxide and oxidative stress. Circulation, 108: 530-535.

8. Baynard T, Miller WC \& Fernhall B (2003). Effects of exercise on vasodilatory capacity in endurance- and resistance-trained men. European Journal of Applied Physiology, 89: 69-73.

9. Copeland SK, Mills MC, Lerner JL, Crizer MF, Thompson CW \& Sullivan JM (1996). Hemodynamic effects of aerobic vs resistance exercise. Journal of Human Hypertension, 10: 747-753.

10. Maeda S, Miyauchi T, Kakiyama T, Sugawara J, lemitsu M, Irukayama-Tomobe $\mathrm{Y}$, Murakami H, Kumagai $\mathrm{Y}$, Kuno S \& Matsuda $M$ (2001). Effects of exercise training of 8 weeks and detraining on plasma levels of endothelium-derived factors, endothelin-1 and nitric oxide, in healthy young humans. Life Sciences, 69: 1005-1016.

11. Carlson LA \& Mossfeldt F (1964). Acute effects of prolonged, heavy 
exercise on the concentration of plasma lipids and lipoprotein in man. Acta Physiologica Scandinavica, 62: 51-59.

12. Perseghin G, Price TB, Petersen KF, Roden M, Cline GW, Gerow K, Rothman DL \& Shulman GI (1996). Increased glucose transportphosphorylation and muscle glycogen synthesis after exercise training in insulin-resistant subjects. New England Journal of Medicine, 335: 1357-1362

13. Kenney MJ \& Seals DR (1993). Postexercise hypotension: key features, mechanism, and clinical significance. Hypertension, 22: 653-664.

14. Brandao Rondon MU, Alves MJ, Braga AM, Teixeira OT, Barretto AC, Krieger EM \& Negrao CE (2002). Postexercise blood pressure reduction in elderly hypertensive patients. Journal of the American College of Cardiology, 39: 676-682.
15. Kingwell BA, Sherrad B, Jennings GL \& Dart AM (1997). Four weeks of cycle training increases basal production of nitric oxide from the forearm. American Journal of Physiology, 272: H1070-H1077.

16. Agewall S, Hulthe J, Fagerberg B, Gottfridsson B \& Wikstrand J (2002). Post-occlusion brachial artery vasodilation after ischaemic handgrip exercise is nitric oxide mediated. Clinical Physiology and Functional Imaging, 22: 18-23.

17. Engelke KA, Halliwill JR, Proctor DN, Dietz NM \& Joyner MJ (1996). Contribution of nitric oxide and prostaglandins to reactive hyperemia in the human forearm. Journal of Applied Physiology, 81: 1807-1814.

18. Halliwill JR, Taylor JA \& Eckberg DL (1996). Impaired sympathetic vascular regulation in humans after acute dynamic exercise. Journal of Physiology, 495: 279-288. 